\title{
Application of Hückel Molecular Orbital Theory (HMO) on Hetero-Conjugated Molecule, 3-Aminopropenal
}

\author{
BILJANA B. ARSIC ${ }^{1 *}$, JOVICA V. UROSEVIC ${ }^{2}$, MIROSLAV M. MITIC ${ }^{2}$ \\ ${ }^{1}$ Department of Mathematics, Faculty of Sciences and Mathematics, University of Niš, Visegradska 33, 18000 Niš, \\ Republic of Serbia. \\ ${ }^{2}$ Chemical-technological school “Bozidar Dorđevic Kukar”, Vlajkova 94, 16000 Leskovac, Republic of Serbia
}

Abstract: Very often the application of quantum mechanics into chemistry represents a challenging task for chemistry students. However, this can be very usual exercise, and we have shown the easiness on the molecule of 3-aminopropenal, which is an interesting example because of the existence of the conjugation system consisting of the carbonyl group, alkenyl system and the lone electronic pair on nitrogen without any symmetry. Coefficients obtained using the Hückel secular determinant were $2.0484,-1.7328,-0.7827,+0.4892$ and +1.5747 .

Keywords: 3-aminopropenal, MO Theory, $\pi$ electronic distribution, quantum mechanics.

\section{Introduction}

There is worldwide problem on the brain drain and insufficient interest in natural sciences, particularly physics and chemistry, which youngsters see as "waste of time and energy". Common trend in teaching is present through the application of different teaching methods, and popularization of natural sciences [1-3].

Covalent bond formation between two or more atoms in the molecule can be explained using valence-structural theory (VS method) and molecular-orbital method (MO-method). MO method investigates the molecule as an entity, thus on the same way as an atom, with the difference of the motion of electrons under the influence of more nuclei, i.e. in molecular orbitals.

Schrodinger equation (1) and its modified form (2) are used for the calculation of the energy of the electron in molecular orbitals.

$$
\begin{aligned}
& \mathcal{\varkappa}^{\wedge} \psi=E \psi \\
& \left(-\frac{\hbar^{2}}{2 m} \nabla^{2}+V\right) \psi=E \psi
\end{aligned}
$$

In equations (1) and (2), $\Psi$ is a wave function, $E$ is the energy of the molecular orbital, $\mathcal{\varkappa}$ is a quantum mechanical operator, which represents the total energy of the system. Usually, it is scary for students, but it only represents the group of the operations written in a shorter form that must be performed over the function $\psi$ :

$$
\varkappa=-\frac{h^{2}}{8 \pi^{2} m} \nabla^{2}+V
$$

Unfortunately, total solutions of the wave equation are today only possible for the simplest case of the hydrogen atom.

In case of the molecule with $n$ electrons and $\eta$ nuclei with the charge $Z_{\mu}$, the equation for Hamiltonian becomes:

$$
\mathcal{K}=\sum_{i=1}^{n}\left(-\frac{\hbar^{2}}{2 m_{i}} \nabla^{2}-\sum_{\mu=1}^{n} \frac{z_{\mu} e^{2}}{r_{i \mu}}+\sum_{(i<j)} \frac{e^{2}}{r_{i j}}\right)
$$

$\overline{\text { *email: Biljana.Arsic@pmf.edu.rs }}$ 
The equation (3) can be simplified by the introduction of Born-Oppenheimer approximation and by the omission of terms related completely to nuclei.

\section{Materials and methods}

The inability of the accurate solution of the wave equation for multiatomic molecules gave different approximate approaches. One of the best is a linear combination of atomic orbitals (LCAO) $[4,5]$, and Huckel [6] posted a theory suitable for the determination of the electronic structure of olefinic and aromatic hydrocarbons (Hückel molecular orbital theory (HMO theory)) [7]. Hückel introduced approximations into modified wave equation, and they are:

Coulomb integral $(\alpha)$

For carbon $i$,

$$
H_{i i}=\int \Phi_{i} \varkappa \Phi_{i} d \tau=\alpha
$$

where $H_{i i}$ is a matrix of elements which appear on the diagonal of the determinant. These terms represent the energy of the interactions of electrons in the isolated $2 p_{z}$ orbital with its own nucleus [8].

Integral of the resonance $(\beta)$ represents the energy of the interaction of electrons of two nuclei at the same time, made by the overlapping of $2 p_{z} A O$ on the observed atoms [8].

$$
H_{i j}=\int \Phi_{i} \mathcal{H} \Phi_{j} d \tau=\beta \text { or } 0
$$

In this case, the interaction should be quite similar for all $i j$ pairs because all $\sigma$-bond distances are approximately the same and all $2 p_{z} A O$ share the same node plane. Therefore, it is not incorrect to put approximation that these $H_{i j}$ terms are equal to the same value $\beta$. If centers $i$ and $j$ are not directly connected, the internuclear distance should be longer and the interaction energy represented with $H_{i j}$ should be significantly smaller. Then, the approximation is performed to make these terms zero in order to simplify the calculation [8].

Overlapping integrals $\left(S_{i j}\right)$ represent the measure of the overlapping of atomic $p$ orbitals.

$$
\begin{aligned}
S_{i j}=\int \Phi_{i} \Phi_{j} d \tau & =1(\mathrm{i}=\mathrm{j}) \\
& =0(\mathrm{i} \neq \mathrm{j})
\end{aligned}
$$

Introducing all three approximations, secular equations and determinants are becoming simpler and easier for solving, even for the more complex systems:

$$
\begin{aligned}
& \mathrm{C}_{1}(\alpha-\varepsilon)+\mathrm{C}_{2} \beta_{12}+\mathrm{C}_{3} \beta_{13}+\ldots+\mathrm{C}_{\mathrm{n}} \beta_{1 \mathrm{n}}=0 \\
& \mathrm{C}_{1} \beta_{21}+\mathrm{C}_{2}(\alpha-\varepsilon)+\mathrm{C}_{3} \beta_{23}+\ldots+\mathrm{C}_{\mathrm{n}} \beta_{2 \mathrm{n}}=0 \\
& \mathrm{C}_{1} \beta_{31}+\mathrm{C}_{2} \beta_{32}+\mathrm{C}_{3}(\alpha-\varepsilon)+\ldots+\mathrm{C}_{\mathrm{n}} \beta_{3 \mathrm{n}}=0 \\
& \vdots \vdots \\
& \mathrm{C}_{1} \beta_{\mathrm{n} 1} \quad+\ldots+\mathrm{C}_{\mathrm{n}}(\alpha-\varepsilon)=0
\end{aligned}
$$

$$
\left|\begin{array}{ccccc}
\alpha-\varepsilon & \beta_{12} & \beta_{13} & \cdots & \beta_{1 n} \\
\beta_{21} & \alpha-\varepsilon & \beta_{23} & & \beta_{2 n} \\
\beta_{31} & \beta_{32} & \alpha-\varepsilon & & \vdots \\
\vdots & & & \ddots & \\
\beta_{n 1} & & \cdots & & \alpha-\varepsilon
\end{array}\right|=0
$$

If we develop the determinant, we are getting $n^{\text {th }}$ level of the secular polynomial $P_{S}$ which possesses real roots of the shape:

$$
\begin{aligned}
& \left(\alpha-\varepsilon_{j}\right)=m_{j} \beta(\mathrm{j}=1,2,3, \ldots \mathrm{n}) \\
& \text { and } n \text { connected energy values: } \varepsilon_{\mathrm{j}}=\alpha-\mathrm{m}_{\mathrm{j}} \beta,
\end{aligned}
$$

which are permitted energy values for MO or so-called Hückel eigenvalues.

If a molecule contains a heteroatom $(\mathrm{O}, \mathrm{N}, \mathrm{S})$, the correction must be introduced for $\alpha$ and $\beta$ integrals related to the carbon atom $[9,10]$ : 


$$
\begin{aligned}
& \alpha_{x}=\alpha_{0}+h_{x} \beta_{0} \\
& \beta_{i j}=k_{i j} \beta_{0}
\end{aligned}
$$

In the equations (9) and (10), $\alpha_{0}$ is a standard value, $\beta_{0}$ is a standard bond integral, $h_{x}$ is the empirical constant without unit, which is characteristic for $X$, and depends on the difference in electronegativity of $\mathrm{X}$ and carbon $[9,10]$. One of the ways to get the parameter $h_{x}$ is the use of Pauling value for the electronegativity $(\chi): h_{x}=k\left(\chi_{x}-\chi_{C}\right)$; it is the common practice to get $k$ very close to one (Table 1S). $k_{i j}$ is being taken as one for all normal C-C bonds in polyenes.

Coefficients $C_{j i}$ are fractions of atomic orbitals in molecular MO:

$$
\Psi_{j}=C_{j 1} \Phi_{1}+C_{j 2} \Phi_{2}+C_{j 3} \Phi_{3}+\ldots+C_{j n} \Phi_{n}(\mathrm{j}=1,2,3, \ldots, \mathrm{n})
$$

and for each of these $\Psi_{j}$ (or eigenfunctions) it exists the appropriate root $m_{j}$ (or $x_{j}$ ) which is related to the energy of the electron in that particular MO. For each $\Psi_{j}$ there is a set of secular equations from which it is possible to get the secular determinant. The problem is now the incorporation of each root into the corresponding set of secular equations, and the solving of the set of coefficients $C_{j i}$ for each $\Psi_{j}$. This gives us a description of the behavior (spatial distribution) of electrons in each MO. They can be obtained from secular equations:

$$
\begin{aligned}
& \mathrm{C}_{\mathrm{j} 1}{ }^{2}+\mathrm{C}_{\mathrm{j} 2}{ }^{2}+\ldots+\mathrm{C}_{\mathrm{ji}}{ }^{2}=1 \\
& \mathrm{C}_{\mathrm{j} 1}(\alpha-\varepsilon)+\mathrm{C}_{\mathrm{j} 2} \beta=0 \\
& \mathrm{C}_{\mathrm{j} 1} \beta+\mathrm{C}_{\mathrm{j} 2}(\alpha-\varepsilon)+\mathrm{C}_{\mathrm{j} 3} \beta=0 \\
& \vdots \\
& \mathrm{k}_{\mathrm{ji}} \beta \mathrm{C}_{\mathrm{ji}-1}+(\alpha-\varepsilon) \mathrm{C}_{\mathrm{ji}}=0
\end{aligned}
$$

If we know energies of molecular orbitals $(E)$, we can calculate total energies of the electrons, resonance energies, etc., and use coefficients $\mathrm{C}_{\mathrm{ji}}$ molecular-orbital indexes.

There are several very useful parameters:

Electronic density $(q)$ represents the probability of finding the electrons on particular atoms in the molecular orbital, and it can be calculated according to the equation (12):

$$
q=\sum_{i}^{o c} n_{i} C_{j i}^{2}
$$

where $n_{i}$ is the number of electrons in MO, $C_{j i}{ }^{2}$ electronic density on atoms, and $i$ occupied orbital.

Electronic charge $(\xi)$ is the charge of the atom and represents the difference of formal charge $(Z)$ [11] and electronic density $(q)$ :

$\xi=Z-q, Q=1$ for a neutral carbon atom

$Z=($ number of the group in the Periodic table)-(number of common electrons/2+number of lone electrons)

$Z(O)=1, Z(N)=1, Z(C)=1$

$\rho$ and $N$ bond order

$\rho$ is a $\pi$ bond order which determines the fraction of the electronic density which belongs to a particular bond.

$$
\rho_{X Y}=\sum_{i}^{o c} n_{i} C_{X} C_{Y}
$$

In the equation (13), $X$ and $Y$ are neighboring atoms, $C$-coefficient, $i$-bonded orbital, $n_{i}$-number of atoms in the bonded orbital. $N$ is the total bond order and represents the sum of $\sigma$ and $\pi$ bonds.

$$
N_{X Y}=1+\rho_{X Y}
$$

where 1 stands for $\sigma$ bond, $\rho_{X Y}$ is $\pi$ bond order, $X$ and $Y$ are neighboring atoms.

Index of free valence $(F)$ represents the reactivity of the center:

$$
F_{X}=N_{\max }-N_{X Y}
$$

where $\sigma$-bond orders are excluded from the consideration because it is assumed that any carbon in $\pi$ system is $s p^{2}$ hybridized and completely $\sigma$-bonded to three other atoms. Regarding reactivity, this is 
understandable because molecules which contain $\pi$ usually react firstly through electrons the most available or electrons of the highest energy, i.e. $\pi$-electrons. $N_{\max }$ can be obtained by the observation of the central atom in the hypothetic molecule of trimethylmethane with a value $\sqrt{3}$ or 1.732 and represents the maximum number of $\pi$ bonds.

$$
\begin{aligned}
& N_{r}=\sum \rho \text {-total sum of bond order around the observed center } \\
& \qquad F_{X}=1.732-\sum \rho
\end{aligned}
$$

\section{Results and discussions}

Semiempirical HMO theory was applied for the determination of $E, \psi$ and $M O$ indexes of the acyclic conjugated compound represented with the structural formulae:

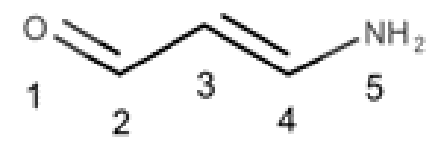

Firstly, Huckel secular determinant was established:

$$
\begin{aligned}
& \alpha_{(} \dot{O}_{)}=\alpha_{0}+1 \beta_{0} \\
& \beta_{(} \dot{O}_{)}=1 \beta_{0} \\
& \alpha\left(\ddot{N}_{)}=\alpha_{0}+1.5 \beta_{0}\right. \\
& \beta(\ddot{N})=0.8 \beta_{0} \\
& \left|\begin{array}{ccccc}
(x+1) & 1 & 0 & 0 & 0 \\
1 & x & 1 & 0 & 0 \\
0 & 1 & x & 1 & 0 \\
0 & 0 & 1 & x & 0.8 \\
0 & 0 & 0 & 0.8 & (x+1.5)
\end{array}\right|=0
\end{aligned}
$$

By the development of the determinant, we are getting polynomial which solutions are zeros of the polynomial $\left(\mathrm{x}_{1} \ldots \mathrm{n}\right)$ (Table 1$)$.

$\mathrm{x}^{5}+2.5 \mathrm{x}^{4}-2.14 \mathrm{x}^{3}-7.14 \mathrm{x}^{2}-0.72 \mathrm{x}+2.14=0$

For all $x$ of the polynomial, the energy of the molecular orbital $(E)$ was calculated (Table 1).

Table 1. Values of $x$ and appropriate $E$

\begin{tabular}{|c|c|c|}
\hline $\mathbf{x}$ & Value & $\mathbf{E}=\boldsymbol{\alpha}-\mathbf{x} \boldsymbol{\beta}$ \\
\hline $\mathbf{1}$ & -2.0484 & $\mathrm{E}_{1}=\alpha+2.0484 \beta$ \\
\hline $\mathbf{2}$ & -1.7328 & $\mathrm{E}_{2}=\alpha+1.7328 \beta$ \\
\hline $\mathbf{3}$ & -0.7827 & $\mathrm{E}_{3}=\alpha+0.7827 \beta$ \\
\hline $\mathbf{4}$ & +0.4892 & $\mathrm{E}_{4}=\alpha-0.4892 \beta$ \\
\hline $\mathbf{5}$ & +1.5747 & $\mathrm{E}_{5}=\alpha-1.5474 \beta$ \\
\hline
\end{tabular}

$E_{1}, E_{2}, E_{3}$ are energies of the bonded orbitals, and $E_{4}, E_{5}$ are energies of anti-bonded orbitals (Figure 1).

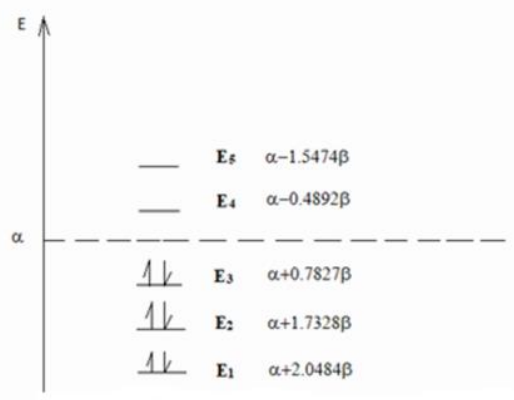

Figure 1. Diagram of the energy levels for the investigated molecule 
Filling of the molecular orbitals (MO) with electrons is almost the same as the filling of atomic orbitals and the same principles are followed.

By posting the analogous secular equations for each value of the zeros of the polynomial $(x)$ (Table $1)$, the coefficients $\left(a_{1 \ldots 5}\right)$ (Table 2$)$ can be calculated.
$\mathrm{a}_{1}^{2}+\mathrm{a}_{2}^{2}+\mathrm{a}_{3}^{2}+\mathrm{a}_{4}^{2}+\mathrm{a}_{5}^{2}=1$
$(\mathrm{x}+1) \mathrm{a}_{1}+\mathrm{a}_{2}=0$
$a_{1}+a_{2} x+a_{3}=0$
$a_{2}+a_{3} x+a_{4}=0$
$\mathrm{a}_{3}+\mathrm{a}_{4} \mathrm{x}+0.8 \mathrm{a}_{5}=0$
$0.8 \mathrm{a}_{4}+(\mathrm{x}+1.5) \mathrm{a}_{5}=0$

Table 2. Values for the coefficients $(a)$ for each value $(x)$

\begin{tabular}{|c|c|c|c|c|c|}
\hline $\mathbf{X}$ & $\mathbf{a}$ & $\mathbf{a}$ & $\mathbf{a}$ & $\mathbf{a}$ & $\mathbf{a}$ \\
\hline $\mathbf{x} 1$ & +0.3387 & +0.3550 & +0.3886 & +0.44095 & +0.6433 \\
\hline $\mathbf{x} 2$ & +0.63096 & +0.4624 & +0.1702 & -0.1674 & -0.5754 \\
\hline $\mathbf{X} 3$ & +0.5337 & -0.11595 & -0.6244 & -0.3728 & +0.4158 \\
\hline $\mathbf{x} 4$ & +0.3963 & -0.5911 & -0.1078 & +0.6438 & -0.2589 \\
\hline $\mathbf{X} 5$ & +0.2118 & -0.5453 & +0.6469 & -0.4737 & +0.1232 \\
\hline
\end{tabular}

Knowing coefficients $a_{1 \ldots 5}$ (Table 2) the explicit forms of wave functions $\psi$ can be written:

$\Psi_{1}=0.3387 \Phi_{1}+0.3550 \Phi_{2}+0.3886 \Phi_{3}+0.44095 \Phi_{4}+0.6433 \Phi_{5}$

$\Psi_{2}=0.63096 \Phi_{1}+0.4624 \Phi_{2}+0.1702 \Phi_{3}-0.1674 \Phi_{4}-0.5754 \Phi_{5}$

$\Psi_{3}=0.5337 \Phi_{1}-0.11595 \Phi_{2}-0.6244 \Phi_{3}-0.3728 \Phi_{4}+0.4158 \Phi_{5}$

$\Psi_{4}=0.3969 \Phi_{1}-0.5911 \Phi_{2}-0.1078 \Phi_{3}+0.6438 \Phi_{4}-0.2589 \Phi_{5}$

$\Psi_{5}=0.2118 \Phi_{1}-0.5453 \Phi_{2}+0.6469 \Phi_{3}-0.4737 \Phi_{4}+0.1232 \Phi_{5}$

MO indexes can be calculated from equations and coefficients $a_{1 \ldots 5}$, and they are written in Tables $2 \mathrm{~S}$ and $3 \mathrm{~S}$.

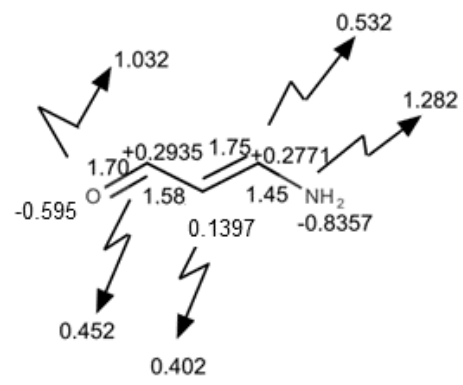

Figure 2. $\pi$ electronic distribution in

3-aminopropenal

On the basis of the obtained parameters, the reactivity of compounds can be calculated and the correlation with some properties of the molecule $(1, \mu$, etc. $)$.

\section{Conclusions}

By the application of semi-empirical HMO theory, approximative values of energies of molecular orbitals were found, explicit values of wave functions were presented, and MO indexes were calculated in an acyclic conjugated compound with two heteroatoms $(\mathrm{O}, \mathrm{N})$ in positions $\alpha$ and $\omega$. Calculated theoretical parameters can be used in correlation with experimental data of the series of similar molecules.

Acknowledgments: We would like to thank Dr. Gordana Stojanović and Dr. Nikola Nikolić, both of them from The Department of Chemistry, Faculty of Sciences and Mathematics, University of Niš, the 
Republic of Serbia for their valuable suggestions during the creation of the manuscript. Dr Biljana Arsic would like to thank Ministry of Science, Education and Technological Development of the Republic of Serbia for the financial support for this research (Project No. 174007).

\section{References}

1. HRAST S., FERK SAVEC V., The integration of submicroscopic representations used in chemistry textbook sets into curriculum topics, Acta Chim. Slov., 64, 2017, p. 959-967.

2. LOGAR A., PEKLAJ C., FERK SAVEC V., Effectiveness of student learning during experimental work in primary school, Acta Chim. Slov., 64, 2017, p. 661-671.

3. RODIC D.D., RONCEVIC T.N., SEGEDINAC M.D., The accuracy of macrosubmicro symbolic language of future chemistry teachers, Acta Chim. Slov., 65, 2018, p. 394-400.

4. LENNARD-JONES J.E., The electronic structure of some diatomic molecules, Trans. Faraday Soc., 25, 1929, p. 668-686.

5. MULLIKEN R.S., Electronic structures of polyatomic molecules and valence VI. On the method of molecular orbitals, J. Chem. Phys., 3, 1935, p. 375-378.

6. HUCKEL E., Quantentheoretische Beitrage zum Benzolproblem, I. DieElektronenkonfiguration des Benzols und verwandter Verbindungen, Z. Phys., 70, 1931, p. 204-286.

7. TRINAJESTIĆ N., Molekularne orbitale u kemiji, Skolska knjiga, Zagreb, 1974, p. 21 (in Croatian).

8. YATES K., Huckel molecular orbital theory, Academic Press, New York, 1978.

9. COULSON C.A. Valence, Oxford Univ. Press, London and New York, 1952, p. 242.

10. WHELAND G.W., PAULING L., A quantum mechanical discussion of orientation of substituents in aromatic molecules, J. Am. Chem. Soc., 57, 1935, p. 2086-2095.

11.TRINAJESTIC N. Molekularne orbitale u kemiji, Skolska knjiga, Zagreb, 1974, p. 46 (in Croatian).

$\overline{\text { Manuscript received: } 24.10 .2019}$

\section{Supplementary Material}

Table 1S. HMO parameters for heteroatoms (X)

\begin{tabular}{|c|c|c|c|}
\hline Atom $(\mathrm{X})$ & $\mathbf{h}_{\mathbf{X}}$ & bond $(\mathrm{C}-\mathrm{X})$ & $\mathbf{k}_{\mathrm{C}-\mathbf{x}}$ \\
\hline$N$ & 0.5 & $\mathrm{C}-\mathrm{N}$ & 0.8 \\
\hline$\ddot{\boldsymbol{N}}$ & 1.5 & C- $-\ddot{N}$ & 0.8 \\
\hline$\dot{0}$ & 1.0 & $\mathrm{C}=\mathrm{O}$ & 0.8 \\
\hline$\ddot{0}$ & 2.0 & $\mathrm{C}=\mathrm{O}$ & 1.0 \\
\hline
\end{tabular}

\begin{tabular}{cccc}
\multicolumn{5}{c}{ Table 2S. MO indexes } & \\
\hline Atom number & $\mathbf{q}$ & $\boldsymbol{\xi}$ & $\mathbf{F}$ \\
\hline $\mathbf{1}$ & 1.5953 & -0.5953 & 1.032 \\
\hline $\mathbf{2}$ & 0.7065 & 0.2935 & 0.452 \\
\hline $\mathbf{3}$ & 1.1397 & -0.1397 & 0.402 \\
\hline $\mathbf{4}$ & 0.7229 & 0.2771 & 0.532 \\
\hline $\mathbf{5}$ & 1.8357 & -0.8357 & 1.282 \\
\hline
\end{tabular}

Table 3S. MO indexes

\begin{tabular}{ccc}
\hline & $\rho$ & $\mathrm{N}$ \\
\hline Neighboring atoms & 0.70 & 1.70 \\
\hline $1 / 2$ & 0.58 & 1.58 \\
\hline $2 / 3$ & 0.75 & 1.75 \\
\hline $3 / 4$ & 0.45 & 1.45 \\
\hline
\end{tabular}

